\title{
Análisis documental relacionado con la educación continua como eje integrador de las competencias del currículo universitario
}

\section{Documentary analysis related to continuing education as the integrating axis of the competences of the university curriculum}

\author{
Luis Danilo Flores Rivera*1 \\ Idaniflores77@gmail.com \\ Carlos Fernando Meléndez Tamayo* \\ cmelendez77@uta.edu.ec \\ Manuel Morocho Amaguaya** \\ mmorocho_a@espoch.edu.ec \\ * Universidad Técnica de Ambato (UTA), Ambato, Ecuador \\ ** Escuela Superior Politécnica de Chimborazo (ESPOCH), Riobamba, Ecuador
}

\section{Resumen:}

El artículo presenta a la educación continua como un recurso fundamental y eficiente del currículo universitario y su mecanismo innovador es el empleo de las Tecnologías de la Información y Comunicación en todas las modalidades de estudio, metodologías y/o procesos de enseñanza-aprendizaje. El estudio tiene como objetivos analizar a la educación continua como eje integrador de las competencias del currículo universitario y difundir resultados oficiales del proceso. Para lo cual, se realizó un análisis conceptual y un análisis descriptivo cuantitativo con un diseño no experimental longitudinal. La investigación registró datos de una Institución de Educación Superior desde el año 2014

\begin{abstract}
:
This article presents continuing education as a fundamental and efficient resource of the university curriculum, being its most innovative mechanism the use of Information and Communication Technologies in all study modalities, methodologies and / or teaching-learning processes. This study aims to analyze continuing education as an integrating axis of the competences in the university curriculum and to disseminate official results of the process. To this end, a conceptual analysis and a quantitative descriptive analysis with a longitudinal non-experimental design were conducted. We collected data from a Higher Education Institution from 2014 to 2019 with a total of 190 courses with 9276
\end{abstract}

1 Dirección para correspondencia (correspondence address):

Luis Danilo Flores Rivera. Educación Continua a Distancia y Virtual, Avenida Los Chasquis y Río Guayllabamba, 180206 Ambato (Ecuador) 
Análisis documental relacionado con la educación continua como eje integrador de las competencias del currículo universitario

luis Danilo Flores Rivera, Carlos Fernando Meléndez Tamayo y Manuel Morocho

AMAGUAYA

hasta el 2019 con un total de 190 cursos con 9276 personas capacitadas y puntualmente el último año con 46 cursos y 1559 participantes. Los resultados del estudio evidencian un crecimiento en el número de cursos, horas y participantes al proceso formativo continuo. Se identifican tendencias actuales de la educación continua vinculada a las TIC; así como requerimientos a otros tipos de programas curriculares. Finalmente el análisis establece el beneficio de la educación continua en la actualización, capacitación, formación y perfeccionamiento de competencias; además la oferta de alternativas que se ajusten a la demanda y requerimientos de los participantes para renovar nuevas competencias sea en el campo académico o laboral.

\section{Palabras claves:}

Educación permanente; formación; habilidad; plan de estudios universitarios; proceso de aprendizaje. people (specifically for last year 46 courses and 1559 participants were included). The results show an increase in the number of courses, hours and participants in the continuous training processes. Current trends in continuing education linked to ICT are identified; as well as requirements for other types of curricular programs. Finally, the analysis establishes the benefits of continuous education when it comes to updating, training and improving skills. Besides, this study also reports an increase in the offer of alternatives required by the participants to update their competencies, whether in academic or labor fields.

\section{Keywords:}

Lifelong education; training; skills; university curriculum; learning processes.

\section{Résumé:}

L'article présente la formation continue comme une ressource fondamentale et efficace du programme universitaire et son mécanisme innovant est l'utilisation des technologies de l'information et de la communication dans toutes les modalités d'étude, les méthodologies et / ou les processus d'enseignement-apprentissage. Les objectifs de l'étude sont d'analyser la formation continue en tant qu'axe d'intégration des compétences du curriculum universitaire et de diffuser les résultats officiels du processus. Pour cela, une analyse conceptuelle et une analyse quantitative descriptive avec un design longitudinal non expérimental ont été réalisées. La recherche a enregistré les données d'un établissement d'enseignement supérieur de 2014 à 2019 avec un total de 190 cours avec 9276 personnes formées et ponctuellement la dernière année avec 46 cours et 1559 participants. Les résultats de l'étude montrent une augmentation du nombre de cours, d'heures et de participants au processus de formation continue. Les tendances actuelles de la formation continue liée aux TIC sont identifiées, ainsi que les besoins pour d'autres types de programmes d'études. Enfin, l'analyse établit l'avantage de la formation continue dans la mise à jour, la formation, la formation et l'amélioration des compétences, ainsi que l'offre d'alternatives qui s'adaptent à la demande et aux exigences des participants pour renouveler de nouvelles compétences, que ce soit dans le domaine académique ou professionnel.

\section{Mots-clés:}

Formation continue; formation; compétence; programme universitaire; processus d'apprentissage.

Fecha de recepción: 15-02-2020

Fecha de aceptación: 26-11-2020 


\section{Introducción}

La educación continua es el eje esencial e integrador de las competencias del currículo, que posibilita la oportunidad e interés de la investigación académica en los distintos modelos de enseñanza y perfiles universitarios que desarrollan competencias profesionales (Junta de Andalucía, 2015, Chanta-Martínez, 2017).

El proceso educativo, en la actualidad, se percibe como un proceso continuo y las Tecnologías de la Información y Comunicación (TIC), proveen un valor agregado a la modalidad. La universidad con el diseño de diferentes cursos ha impulsado la participación de estudiantes y profesionales que desean fortalecer sus conocimientos y competencias, conllevando a un currículo competitivo, adaptado a las necesidades empresariales y de la sociedad actual.

Las competencias específicas o de especialidad del currículo universitario evolucionan rápidamente ante el cambio tecnológico y la transmisión de la información. La gestión académica universitaria es la encargada de manejar las alternativas que sean flexibles y se ajusten al proceso reglamentado. Es aquí donde se hace presente la educación continua como un sistema referencial para actualizar e integrar las competencias necesarias para el desarrollo productivo y tecnológico.

Los objetivos del presente estudio son un análisis documental de la educación continua como eje integrador de las competencias del currículo universitario que se establecen en el contexto académico y laboral. Los conceptos que se enfatizan son "Educación continua", "Importancia de la educación continua", "Capacitación", "Competencias" y "Currículo universitario". El alcance de los conceptos es en todo el artículo debido a su acepción en el escenario académico y profesional, para el caso se revisan estudios, proyectos y experiencias de aprendizaje. Otro objetivo, es difundir resultados oficiales del proceso de educación continua que lleva a cargo la Dirección de Educación Continua a Distancia y virtual (DEaDV) de la Universidad Técnica de Ambato (UTA). La presentación de datos, registra la variación de la oferta, que cada año se ha venido generando en la Institución de Educación Superior (IES) investigada. Se describen historiales estadísticos de la población diana de los cursos ejecutados (número de cursos, horas y participantes matriculados; porcentajes de las áreas en los cursos ofertados), referencias que aportan a la toma de decisiones de la DEaDV; discusión espacio donde se argu- 
Análisis documental relacionado con la educación continua como eje integrador de las competencias del currículo universitario

luis Danilo Flores Rivera, Carlos Fernando Meléndez Tamayo y Manuel Morocho

AMAGUAYA

menta la información; conclusiones que determinan contribuciones al proceso formativo continuo en el currículo universitario.

\section{Marco Teórico}

\section{Educación Continua}

Proceso dinámico que permite la actualización, perfeccionamiento y la formación de conocimientos, habilidades y actitudes; siendo los actores del escenario IES y participantes. Las IES principalmente son Universidades que gestionan y vinculan la educación continua; en cambio, los participantes son estudiantes, profesionales, académicos, personal administrativo y público en general que requieren perfeccionar, mejorar o fortalecer su función experta y/o buscan un crecimiento personal. El procedimiento se apoya de la teoría pedagógica para construir y reforzar el conocimiento; así como cambiar actitudes, conductas y hábitos. La finalidad es mejorar el desempeño profesional y erradicar prácticas no productivas (Puntunet-Bates y Domínguez-Bautista, 2008, Red Universitaria de Educación Continua, 2017, Meléndez-Tamayo y Flores-Rivera, 2018).

Es preciso indicar que a la educación continua, también se la conoce con diferentes nombres como perfeccionamiento, formación técnico profesional, actualización, desarrollo personal entre otras denominaciones, siendo nada más que una estrategia que permita mejorar la educación y el desarrollo personal con el fin de mejorar las prácticas pedagógicas o las competencias profesionales (Cabrera-Núñez, 2016).

En el escenario universitario la educación continua es conocida como la formación formal que ofrecen las IES, y que tiene un valor más significativo que la formación de grado, siendo su propósito capacitar, especializar o actualizar profesionalmente y que esta formación permita una convivencia democrática en la vida social (Sánchez, 2012).

Según el Consejo de Educación Superior del Ecuador, la educación continua:

Hace referencia a procesos de capacitación, actualización y certificación de competencias laborales específicas. Se ejecutan en forma de cursos, seminarios, talleres u otras actividades académicas.

La educación continua es avanzada cuando está dirigida a profesionales y 
desarrollada por expertos de un campo del conocimiento específico.

Esta formación podrá ser organizada a través del sistema de horas y/o créditos. La formación de educación continua no conduce a una titulación de educación superior y solo puede ser homologada mediante el mecanismo de validación de conocimientos.

(Consejo de Educación Superior, 2019, p. 25)

De lo antes mencionado se puede deducir que la educación continua es la modalidad que vincula a estudiantes, profesionales y personas con talante de aprender para ser competitivos; con temáticas de actualización, capacitación, certificación, especialización, formación, entre otras maneras de desarrollo académico, personal y profesional; con el fin de ser productivos en cualquiera de las actividades en que se desempeñen.

Empresas e industrias del sector productivo del país, ven a la educación continua como el vínculo y la oportunidad para encaminarse a las tendencias y exigencias actuales del ámbito industrial y laboral (Andrade-Paco, Nava-Ortega, y Valverde-Núñez, 2009). En un contrasentido los conocimientos que adquieren los partícipes de la educación continua se pueden mermar por cuestiones de tiempo, tendencias, avances tecnológicos entre otros factores. Por tal motivo, la importancia de la educación continua se vuelve trascendental en los procesos de aprendizaje e investigación, que fomentan las competencias académicas y profesionales (Barros-Morales et al., 2019).

\section{Importancia de la educación continua}

La educación continua destaca por su manera dinámica en avances e innovaciones del conocimiento científico y tecnológico en diferentes áreas. La actualización y perfeccionamiento no solo es en profesionales, sino también en el personal docente de las IES. Su modalidad educativa, es flexible a programas curriculares, syllabus y métodos. La innovación es una constante, se relaciona a las necesidades de los sectores productivos entre otros; es una oportunidad de crecimiento y mejoramiento profesional para ser eficaz y eficiente al cambio tecnológico y productivo. Los cursos en línea, están dentro de la transformación y tendencia de la educación continua, por su flexibilidad y oportunidad de generar aprendizajes a cualquier hora del día y en cualquier lugar (Arredondo-Galván, 2012, Izquierdo-García y Schuster-Fonseca, 2009). 
Análisis documental relacionado con la educación continua como eje integrador de las competencias del currículo universitario

luis Danilo Flores Rivera, Carlos Fernando Meléndez Tamayo y Manuel Morocho AMAGUAYA

De la misma forma se destaca la participación de profesionales con amplia experiencia en procesos de educación continua que realizan actividades planificadas y colaborativas con propósitos de crecimiento personal y profesional. Las acciones desarrolladas generan recursos de calidad que benefician a contenidos y programas curriculares, para luego articular una interacción entre los partícipes que proyectarán un análisis crítico y reflexivo que favorece a la construcción de conocimientos significativos que enriquecen la metodología de las áreas de trabajo (Ortega, 2011, Barros-Morales et al., 2019).

Dentro de este orden de ideas, las IES y las organizaciones, están priorizando la importancia de los procesos de educación y formación continua ante el eventual direccionamiento de procesos productivos y tecnológicos. De acuerdo con Pailos (2019) capacitar y desarrollar permite a los empleados y las organizaciones cumplir con el tiempo y los objetivos proyectados para mercados cambiantes, en los que sobreviven los más aptos.

Desde la perspectiva organizacional, la educación continua es considerada para:

- Mejorar las habilidades de los directivos en el desempeño de sus labores diarias; así como la toma de decisiones y la solución de problemas.

- Fortalecer a los profesionales e instruirlos ante la innovación tecnológica y organizacional.

- Robustecer los procesos de transformación organizacional para la obtención de resultados.

- Cumplir con las expectativas de servicio que exige el sector industrial.

(Andrade-Paco, Nava-Ortega, y Valverde-Núñez, 2009)

En efecto, la educación continua promueve la competitividad del campo profesional y potencia las características laborales. Por consiguiente se la considerada como un patrimonio personal, donde la inversión en capacitación es un requerimiento para desempeñar las actividades profesionales y una constante del desarrollo integral con beneficios en corto, mediano y largo plazo (Cejas-Martínez y Acosta, 2012, Jamaica-González, 2016). 
Análisis documental relacionado con la educación continua como eje integrador de las competencias del currículo universitario

luis Danilo Flores Rivera, Carlos Fernando Meléndez Tamayo y Manuel Morocho

AMAGUAYA

\section{Capacitación}

Proceso enseñanza-aprendizaje que facilita el desarrollo de conocimientos, habilidades y destrezas en función de la competencia que determina la operatividad del departamento y/o sección organizacional; esto no sólo implica entendimiento, sino también afectividad para que en conjunto se engrandezca el significado de la experiencia (Puntunet-Bates y Domínguez-Bautista, 2008).

En la figura 1, se presenta un mapa conceptual, con cinco etapas categóricas para que la educación continua y la capacitación tengan el resultado anhelado.

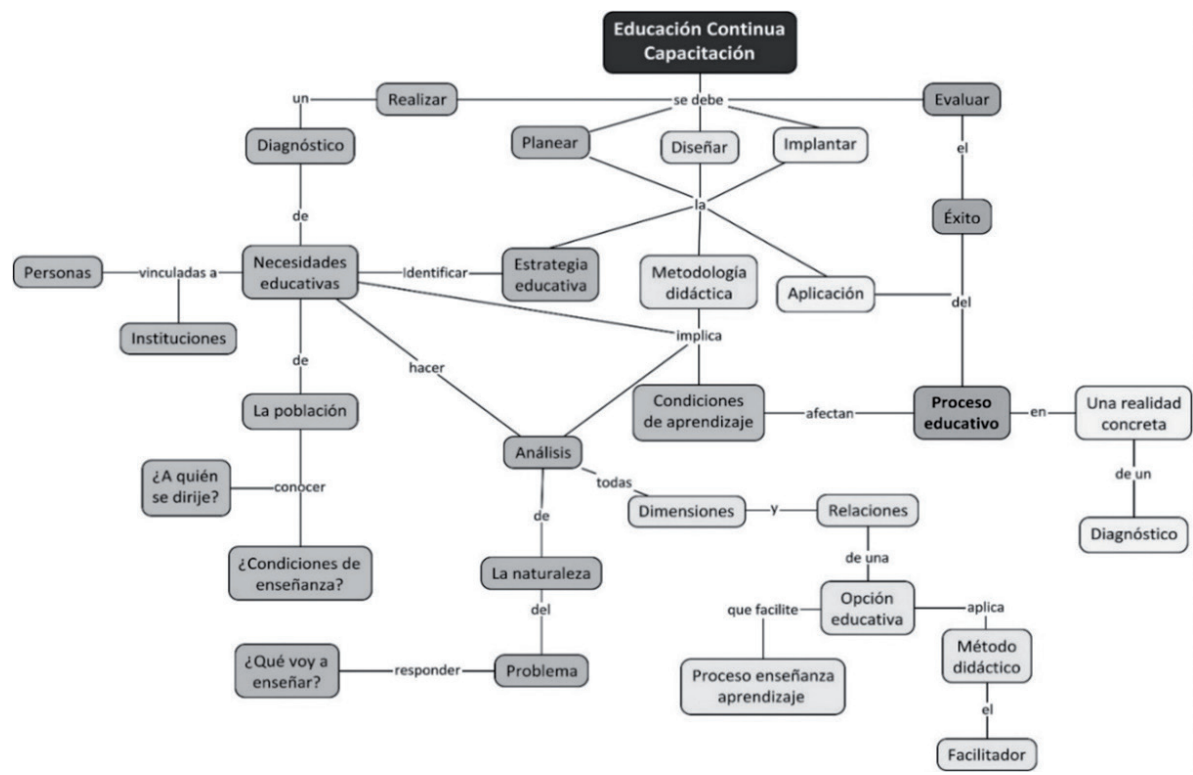

Figura 1. Educación continua en el proceso educativo.

Fuente: Elaboración propia basado en Puntunet-Bates

y Domínguez-Bautista (2008, p. 116).

Se observa que, la capacitación es un factor significativo y de formación permanente en administradores e integrantes de la organización que se benefician mutuamente, mejorando su eficiencia para alcanzar márgenes altos de productividad (Bermúdez-Carrillo, 2015).

Al respecto, Chiavenato (2011), manifiesta que: 
Análisis documental relacionado con la educación continua como eje integrador de las competencias del currículo universitario

luis Danilo Flores Rivera, Carlos Fernando Meléndez Tamayo y Manuel Morocho AMAGUAYA

La capacitación es el proceso educativo de corto plazo, aplicado de manera sistemática y organizada, por medio del cual las personas adquieren conocimientos, desarrollan habilidades y competencias en función de objetivos definidos. La capacitación entraña la transmisión de conocimientos específicos relativos al trabajo, actitudes frente a aspectos de la organización, de la tarea y del ambiente, así como desarrollo de habilidades y competencias. (p. 386)

Es en ese sentido, donde se destaca los procesos de la educación superior "la importancia de abordar capacitación y el perfeccionamiento pedagógicos de los docentes mediante una visión holística, que posibilite su contextualización sobre la base de las necesidades formativas y demandas de aprendizaje" (Arteaga-Linzán, López-Padrón, y Ruiz-Arnaud, 2018, p. 78).

Por otro lado, Rivadeneyra-Espinoza, Rivera-Grados, Sedeño-Monge, López-García y Soto-Vega (2016) exponen que los profesionales de diferentes disciplinas "requieren estar capacitados para promover la generación del conocimiento y lograr el aprendizaje significativo en el alumno" (p. 45).

En efecto, la capacitación en la educación continua, es un modo constante de actualización de conocimientos pedagógicos y metodológicos, que se organizan con el fin de lograr el perfeccionamiento docente (conocimientos, habilidades y actitudes); beneficios de los procesos de enseñanza y la renovación curricular; y una proyección hacia la educación de calidad; que permitan ser competentes ante la complejidad y modernización académica (Flores-Zapata y Aballe-Pérez, 2018, NaresGonzález y Soto-González, 2014).

En relación a la idea anterior, las organizaciones consideran como primordial la capacitación en sus empleados, puesto que mejora sus competencias profesionales y capacidades interpersonales, siendo una oportunidad para su crecimiento al interior de la organización. Sin embargo, se deben considerar estrategias apropiadas como la Capacitación Basada en Competencias (CBC) que puede ser facilitada en el trabajo (proyectos empresariales), sea de forma interna o externa, con su componente de avance la competencia al realizar labores concretas, observables y medibles, que esten esencialmente centradas en el participante o aprendiz (PRODEM, 2004, Universidad ESAN, 2016). 


\section{Competencias}

Cualidad en el desarrollo de actitudes y aptitudes para alcanzar objetivos de aprendizaje relacionados al entorno, en otras palabras se integra al sujeto que aprende al escenario del cual aprende (CEPP y GIZ, 2019). Es conveniente decir, que el análisis de competencia principalmente se vinculará al contexto educativo.

González y Wagenaar (2006) describen a la competencia como una combinación dinámica del conocimiento, comprensión, capacidades y habilidades. La gestión de las competencias es esencial en los programas educativos y se estructuran en varias unidades del curso y son evaluadas en diferentes etapas. Para la consultora PRODEM (2004) la competencia es la "capacidad de articular y movilizar condiciones intelectuales y emocionales en términos de conocimientos, habilidades, actitudes y prácticas necesarias para el desempeño de una determinada función o actividad, de manera eficiente, eficaz y creativa, conforme a la naturaleza del trabajo" (p. 1). La UNESCO (2018) determina a la competencia como "el conjunto de comportamientos socioafectivos y habilidades cognoscitivas, psicológicas, sensoriales y motoras que permiten llevar a cabo adecuadamente un papel, una función, una actividad o una tarea" (p. 39).

Por su parte Beneitone et al. (2007) señalan a la competencia como "una red conceptual amplia, que hace referencia a una formación integral del ciudadano, por medio de nuevos enfoques, como el aprendizaje significativo, en diversas áreas: cognoscitiva (saber), psicomotora (saber hacer, aptitudes), afectiva (saber ser, actitudes y valores)" (p. 36). En relación a lo establecido, las competencias son prioritarias en los sistemas educativos; su selección es claramente un ejercicio social y político vinculado a los valores que cada comunidad social considera imprescindibles para el desarrollo de sus ciudadanos (Gimeno-Sacristán et al., 2008).

A continuación en la figura 2, se ilustra la organización del aprendizaje como un factor clave para alcanzar las competencias. 
Análisis documental relacionado con la educación continua como eje integrador de las competencias del currículo universitario

luis Danilo Flores Rivera, Carlos Fernando Meléndez Tamayo y Manuel Morocho

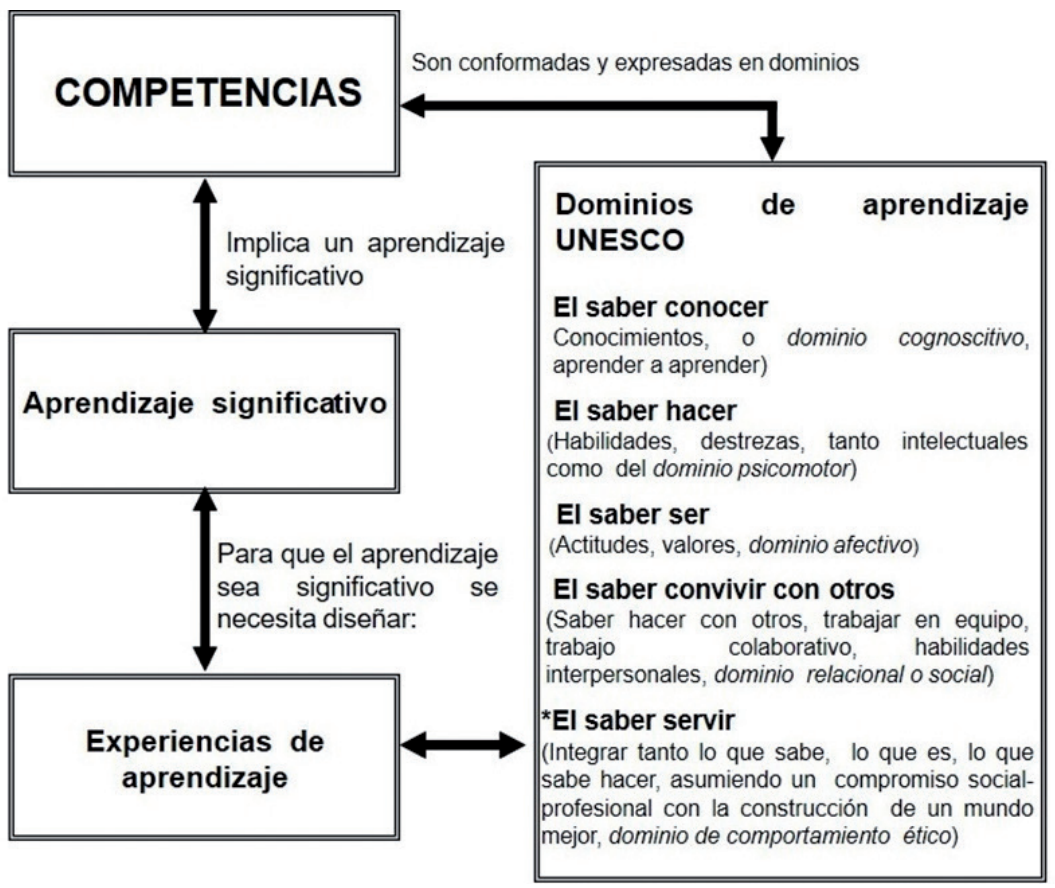

Figura 2. Organización del aprendizaje para alcanzar competencias.

Fuente: (Gómez, 2019)

Una revisión de la educación basada en competencias según EliotLevine (2019) es la que a continuación se muestra:

1. Los estudiantes tienen el poder diario para tomar decisiones importantes sobre sus experiencias de aprendizaje, cómo crearán y aplicarán el conocimiento, y cómo demostrarán su aprendizaje.

2. La evaluación es una experiencia de aprendizaje significativa, positiva y enriquecedora para los estudiantes que brinda evidencia oportuna, relevante y procesable.

3. Los estudiantes reciben apoyo oportuno y diferenciado en función de sus necesidades de aprendizaje individuales.

4. El progreso de los estudiantes se basa en la evidencia de dominio, no en el tiempo en el asiento.

5. Los estudiantes aprenden activamente utilizando diferentes caminos y ritmos variados.

6. Las estrategias para garantizar la equidad para todos los estudiantes están integradas en la cultura, estructura y pedagogía de los institutos y los sistemas educativos. 
7. Las rigurosas expectativas comunes para el aprendizaje (conocimiento, habilidades y disposiciones) son explícitas, transparentes, medibles y transferibles. (p. 3)

De lo planteado, la educación basada en competencias reemplaza al sistema tradicional; impulsa la necesidad de transformar el sistema educativo para que todos los estudiantes puedan aprender con compromiso; apoyados a través de experiencias de aprendizaje auténticas dentro y fuera del aula; la equidad es un objetivo central de la colectividad para el avance de los sistemas educativos basados en competencias; sin dejar de lado lo determinado por Morin (1999) la educación deberá ilustrar el principio de unidad/diversidad en todos los campos (p. 27).

Es en ese sentido que, todos los estudiantes pueden aprender y deben ser desafiados para lograr un aprendizaje complejo con altas expectativas en el que los docentes deben organizar entornos de aprendizaje innovadores en función de las necesidades considerando las diferentes maneras de aprender así como también, los períodos de tiempo (EliotLevine, 2019).

Por lo cual los estudiantes requieren aprender el conocimiento académico, habilidades y prácticas para su aplicación; el aprendizaje ocurre en cualquier momento y en cualquier lugar, este debe ser un aprendizaje complejo, colaborativo e integrado; la transparencia de los intereses de aprendizaje y los resultados de la evaluación es fundamental para crear una cultura de aprendizaje y responsabilidad (EliotLevine, 2019).

Para Grijalva-Verdugo y Lara-Rivera (2019) otro elemento a considerar es la competencia mediática que permite la creación y manipulación de contenido digital considerado como saberes necesarios y relacionados al currículo que integra las TIC. Según Ferrés y Piscitelli (2012) la competencia mediática, es un conjunto de capacidades que se desarroIla para actuar con los medios. Se organiza en seis grandes dimensiones, que se ilustran en la figura 3. 
Análisis documental relacionado con la educación continua como eje integrador de las competencias del currículo universitario

luis Danilo Flores Rivera, Carlos Fernando Meléndez Tamayo y Manuel Morocho

AMAGUAYA

\section{Competencia mediática organizada en seis dimensiones}

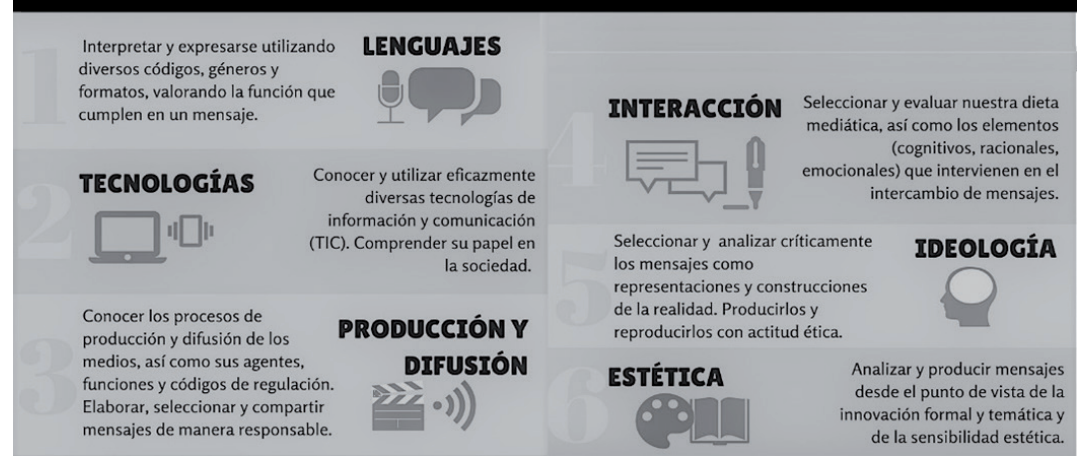

Figura 3. Competencia mediática.

Elaboración: Mateus, 2018 basándose en Ferrés y Piscitelli (2012, pp. 79-81)

La competencia mediática y varias de sus dimensiones, están muy conexas con la competencia digital "el uso creativo, crítico y seguro de las TIC para alcanzar los objetivos relacionados con el trabajo, la empleabilidad, el aprendizaje, el uso del tiempo libre, la inclusión y participación en la sociedad" (Cabero-Almenara, De la Horra-Villacé, y Sánchez-Bolado, 2018, p. 156) (figura 4), con la diferencia que la competencia mediática incluye elementos más expertos. En lo que corresponde al sentido educativo, los sujetos primero tendrían que adquirir la competencia digital para aspirar a una competencia mediática integral (Grijalva-Verdugo y Urrea-Zazueta, 2017).

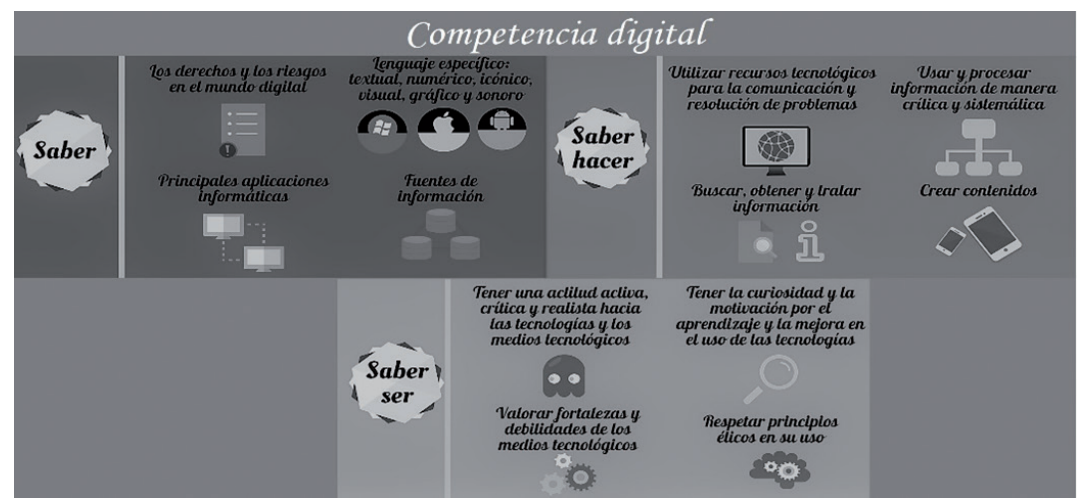

Figura 4. Competencia digital.

Fuente: (Ministerio de Educación y Formación Profesional - Gobierno de España, 2018) 
En concordancia a lo descrito, el enfoque por competencias, asocia al aprendizaje asistido y las competencias mediáticas, que se proyectan en función del currículo por competencias, permitiendo a la persona centrar su mirada en descubrir distintas situaciones de su entorno y ser partícipe de la experiencia (CEPP y GIZ, 2019).

\section{Currículo Universitario}

El currículo aplica una concepción teórico-metodológica a una realidad educativa específica (educación básica, grado, posgrado, entre otras), no es más que un mediador entre la ciencia didáctica y el proceso de enseñanza-aprendizaje.

Por lo antes expuesto, el currículo debe ser universal, adaptarse a diferentes entornos y organizaciones educativas; los educandos, agentes activos, en el proceso de aprendizaje deben sentir comodidad en el ambiente académico. Según Ortiz-Ocaña (2014) el currículo debe comprometer calidad, pertenencia, inclusión, equidad, flexibilidad, multiculturalidad y creatividad para afrontar los problemas e interrogantes que plantea el aprendizaje.

En la figura 5, se presenta las características principales del currículo en la educación superior.

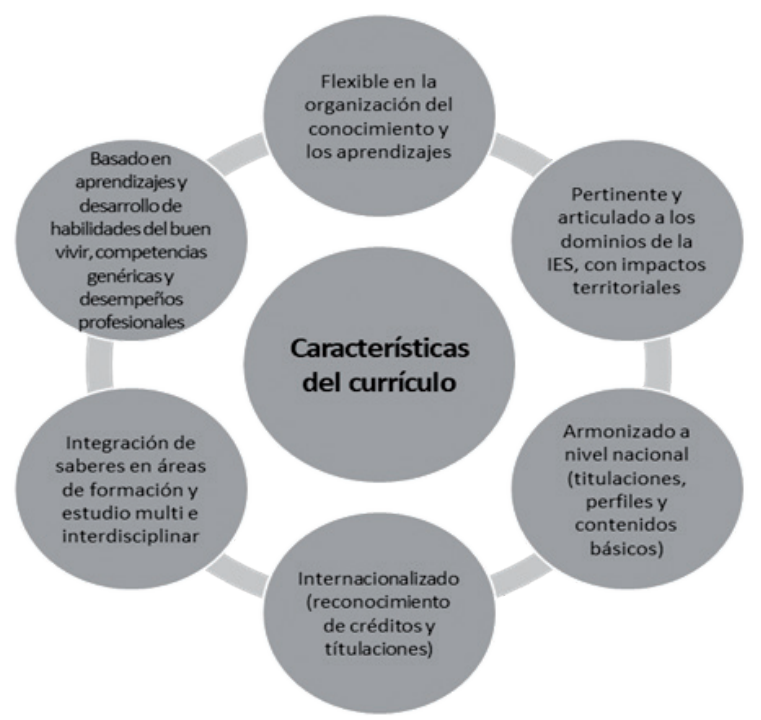

Figura 5. Características del currículo.

Fuente: Elaboración propia basado en Consejo de Educación Superior (2013) 
Análisis documental relacionado con la educación continua como eje integrador de las competencias del currículo universitario

luis Danilo Flores Rivera, Carlos Fernando Meléndez Tamayo y Manuel Morocho AMAGUAYA

El currículo universitario hace referencia al currículo por competencias. La figura 6, muestra un diseño conceptual, con carácter socio formativo del currículo por competencias.

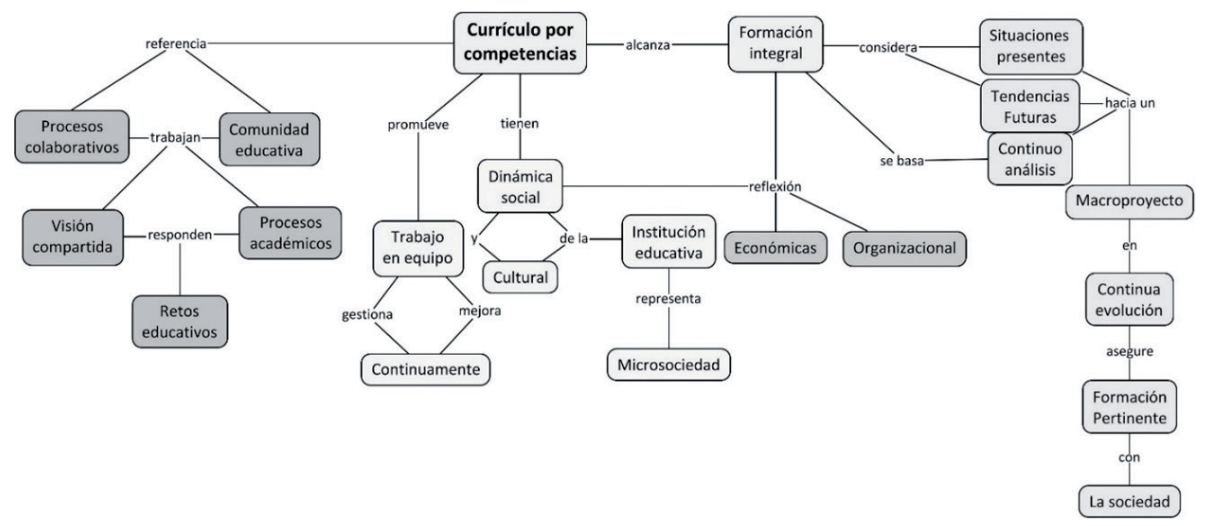

Figura 6. Currículo por competencias socio formativo.

Fuente: Elaboración propia basado en Tobón Tobón, Pimienta

Prieto, y García Fraile (2010, pp. 18-19)

Catalano, Avolio de Cols y Sladogna (2004), Zabalza (2008), Tuning (2007), Tobón et al. (2012), González y González (2008), señalan que el diseño curricular basado en competencias tiene las siguientes características:

- El perfil del proceso formativo es orientado por el conjunto de competencias generales y específicas.

- Las competencias genéricas o específicas se originan de unidades de competencia y estas de elementos de competencias, las cuales se precisan como capacidades de cada uno de los componentes formativos del plan de estudios.

- Adquiere una estructura modular.

- Desarrolla un enfoque integrador en relación a todas sus dimensiones.

- Pretende una integración de capacidades, de contenidos declarativos, procedimentales y actitudinales; de teoría y práctica, de actividades y evaluación.

- Los criterios de aprobación de los distintos módulos se apoyan en los criterios de evaluación establecidos en el logro de desempeños.

- Admite en su desarrollo un enfoque de enseñanza-aprendizaje 
luis Danilo Flores Rivera, Carlos Fernando Meléndez Tamayo y Manuel Morocho

significativo (Huerta-Rosales, Penadillo-Lirio y Kaqui-Valenzuela, 2017).

- Modelo didáctico adaptable, integrador y dinámico que guía el proceso formativo de un programa de una carrera profesional (Huerta, 2014).

A las características señaladas e ilustradas en la figura 6, se pueden acondicionar nuevas propuestas curriculares que incorporan medios innovadores como: flexibilidad, interdisciplinariedad, transversalidad, virtualidad, centrado en el aprendizaje, basado en competencias, personalizado (importancia tutorial), enfocado a la internacionalización, etc. Evidentemente los medios descritos le conceden al currículum universitario la posibilidad de ampliar nuevas formas de enseñanza y de aprendizaje, con una concepción distinta en el proceso evaluativo; aunque tenga similitud al modelo curricular centrado en el paradigma de aprendizaje; con la particularidad de no descuidar la calidad en la enseñanza (Moreno-Olivos 2010).

\section{Método}

El presente artículo, es producto de un análisis conceptual, "método que permite al investigador convertir los conceptos en piezas teóricas precisas para el estudio que quiere llevar a cabo" (Rico, 2004, p. 10), dicho análisis se apoyó del programa CmapTools que permitió modelar mapas conceptuales del estudio efectuado.

Además se realizó un análisis descriptivo cuantitativo con un diseño no experimental longitudinal, que presenta resultados finales desde el año 2014 hasta el 2019 con todos los cursos efectuados por la DEaDV, en las áreas de Educación, TIC, Investigación y Competencias específicas. La población capacitada fue de 9276 personas conformada por estudiantes, personal docente, administrativos y de servicio de la universidad. La información analizada se apoyó del programa Microsoft Excel con resultados presentados en tablas y gráficas con líneas de tendencias para los cursos ejecutados y gráficas de barras para las áreas ofertadas en cada año.

La DEaDV, empleó la plataforma institucional (Sistema informático) para registrar la matrícula de los estudiantes o nómina de estudiantes. El sistema permite migrar la información mediantes tablas de datos que 
Análisis documental relacionado con la educación continua como eje integrador de las competencias del currículo universitario

luis Danilo Flores Rivera, Carlos Fernando Meléndez Tamayo y Manuel Morocho AMAGUAYA

se guardan en hojas de cálculo del programa Excel. La base de datos en Excel es un respaldo de la información. El proceso descrito facilita la minería de datos, que admite el análisis de registros y reportes estadísticos de los cursos ejecutados; asimismo dentro de la base de datos, se consideró informaciones de cursos con organizaciones públicas, privadas y de público en general.

La investigación se fundamentó de la necesidad departamental, estableciendo los siguientes criterios:

a) Análisis de todos los datos consolidados anualmente (población diana) de los participantes a los cursos desarrollados por la Institución, clasificándose por número, número de horas, número de participantes matriculados y porcentajes de las áreas (cursos ofertados);

b) Estimación de la prospectiva institucional, que analiza el interés de la oferta realizada o si hay que modernizar la misma;

c) Determinación del crecimiento de la oferta y la demanda a la participación de los eventos realizados.

Los criterios indicados posibilitan gestionar nuevos cursos y proyectar la disponibilidad del espacio físico, capacidad tecnológica y talento humano especializado en procesos de educación continua. Sin embargo, el análisis se limita a resultados globales con la posibilidad de ampliarse y considerar otras categorías que aportarían mejor a la toma de decisiones de la DEaDV.

\section{Resultados}

En la figura 7, se presenta el desarrollo de los cursos de educación continua ejecutados desde el año 2014 correspondiente a 12 cursos, que se considera como punto base (100\%) hasta el 2019 correspondiente a 46 cursos (383\%), considerada como punto final de la gráfica en línea, evidenciando un crecimiento en cada año muy significativo expresado en porcentajes. Es importante mencionar que la UTA a través de la DEaDV, oferta en las modalidades presenciales, virtuales y b-learning, en cuatro áreas: Tecnologías, Educación, Investigación y Específicas, los cursos son de asistencia y aprobación. El mayor crecimiento año 2018 (400\%) con 49 cursos. Se reitera, que la capacitación fue dirigida a la comunidad universitaria, instituciones públicas, privadas y público general. 


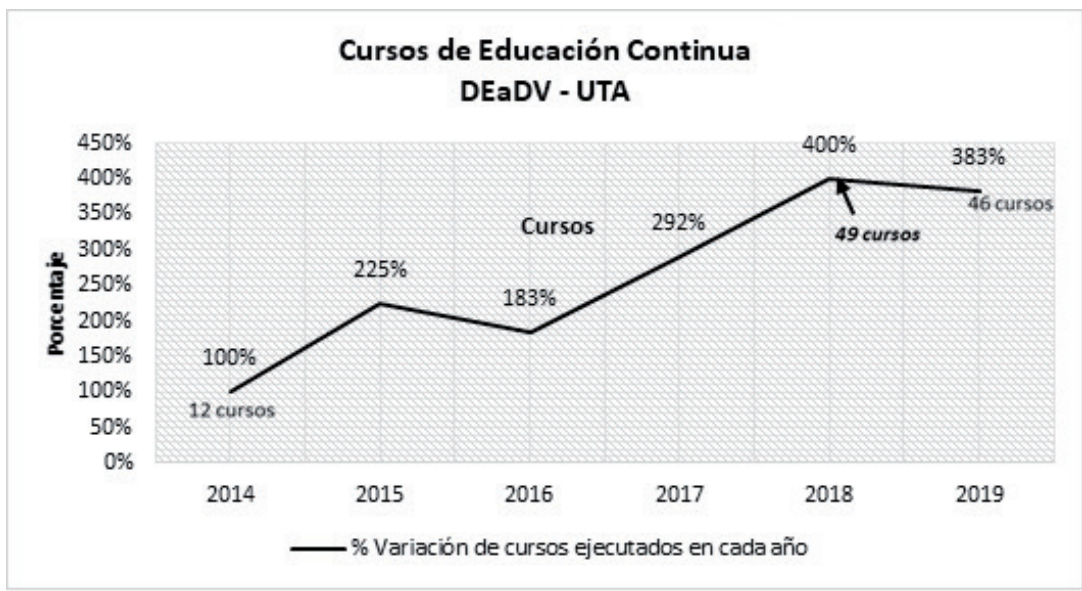

Figura 7. Desarrollo de cursos de educación continua.

Fuente: Elaboración propia basada en datos del sistema informático DEaDV (2020)

En la figura 8, se observa las horas de capacitación desarrolladas desde el 2014 correspondiente a 646 horas, que se considera como punto base (100\%) hasta el 2019 correspondiente a 2060 horas (319\%) considerada como punto final de la gráfica en línea, evidenciando un crecimiento de año a año muy significativo expresado en porcentajes. Asimismo, se aprecia el número de matriculados en el 2014 con 330 participantes siendo el punto base (100\%), y el 2019 con 1559 participantes (472\%) como punto final de la gráfica en línea, mostrando un crecimiento de año a año expresado en porcentajes.

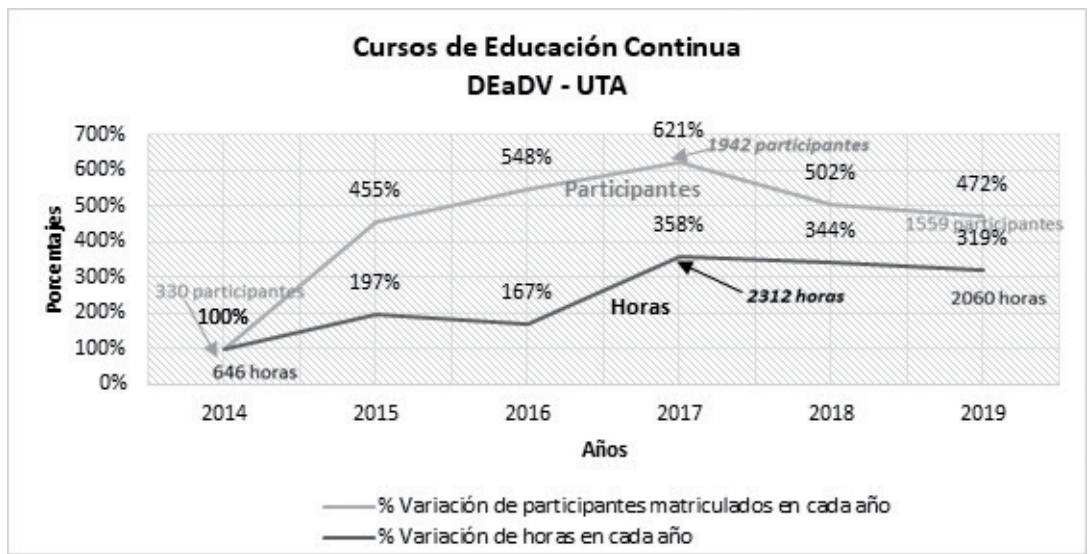

Figura 8. Horas de capacitación versus número de matriculados.

Fuente: Elaboración propia basada en datos del sistema informático DEaDV (2020) 
Análisis documental relacionado con la educación continua como eje integrador de las competencias del currículo universitario

luis Danilo Flores Rivera, Carlos Fernando Meléndez Tamayo y Manuel Morocho AMAGUAYA

En la figura 9, se presenta información de los cursos de educación continua desarrollados en el año 2019, con sus cuatro áreas ofertadas que son Tecnologías (TIC), Docencia (Educación), Investigación y Específicas. Siendo las de mayor demanda el Área de Docencia $31 \%$ y el Área de Investigación 30\% y la de menor demanda el Área Específicas $13 \%$, que responden a necesidades y convenios con instituciones públicas o privadas, así como también con empresas, organizaciones sin fines de lucro en un trabajo cooperativo y colaborativo (DEaDV, 2020).

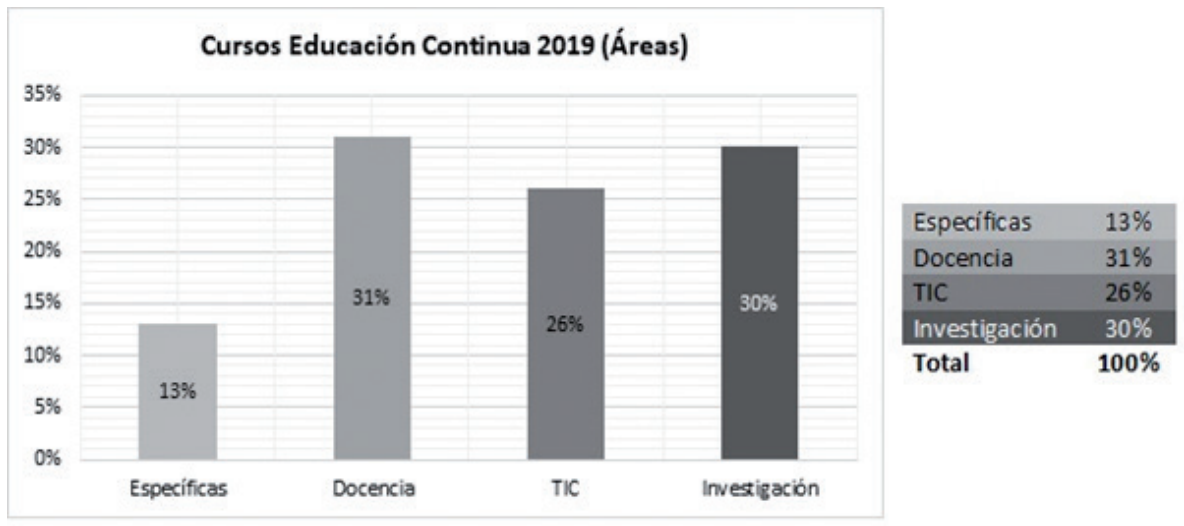

Figura 9. Áreas de los cursos de educación continua 2019.

Fuente: Elaboración propia basada en datos del sistema informático DEaDV (2020)

\section{Discusión}

La educación continua ha incrementado su oferta en sus procesos de formación, debido a los requerimientos de competencias profesionales y el aumento de los modelos de educación (presencial, b-learning y virtual). El empleo del modelo híbrido integrado a las TIC, se destaca como elemento diferenciador que fortalece el proceso de educación continua, permite disponer de una flexibilidad y un ritmo propio para cumplir con las actividades programadas (Meléndez-Tamayo y Flores-Rivera, 2018). De acuerdo con Verdezoto-Rodríguez y Chávez-Vaca (2018) este nuevo escenario de la Educación impone "enseñar a aprender" por parte de los docentes y "aprender a aprender" por parte del estudiantado (p. 87). Por ende, la utilización de plataformas y TIC vienen siendo componentes básicos en los procesos y metodologías de enseñanza-aprendizaje; así 
Análisis documental relacionado con la educación continua como eje integrador de las competencias del currículo universitario

luis Danilo Flores Rivera, Carlos Fernando Meléndez Tamayo y Manuel Morocho

AMAGUAYA

como una oportunidad de incrementar y mejorar conocimientos que resultan provechosos en la educación continua (Verdezoto Rodríguez y Chávez Vaca, 2018, Flores-Rivera y Meléndez-Tamayo, 2017, AreaMoreira y Adell-Segura, 2009).

Los procesos de educación continua del sistema informático DEaDV exponen lo siguiente:

Crecimiento en la oferta de los cursos en los seis años de actividades, resultado observado en la figura 7 . Siendo conveniente, analizar en qué medida los resultados de la oferta de las distintas modalidades de educación continua tiene acogida por parte de los participantes. Sin descartar como estudios posteriores un desglose de las áreas propuestas.

Corresponde decir que la DEaDV consideró las tendencias actuales de la educación continua vinculada a las TIC (Competencias mediáticas, competencias digitales), donde se evidencia que la mayoría de las actividades docentes son «mixtas» o utilizan el modelo «híbrido», donde se reduce la modalidad presencial y se rediseña la modalidad on-line para superar su rol secundario (Valverde-Berrocoso, 2019).

Crecimiento en la oferta de horas de capacitación en los seis años de actividades, resultado observado en la figura 8 , lo que responde a la planificación de los procesos de educación continua por competencias profesionales y a un análisis de las necesidades identificando problemáticas o dificultades a ser mejoradas o potenciadas; dicho cuestionamiento se relaciona con lo precisado por Zapata-Guerrero (2012) que son las expectativas de los potenciales interesados en los currículos o planes formales.

Crecimiento en el número de participantes en los seis años de actividades, resultado observado en la figura 8 . Se comparte lo dicho por Yépez (2019), donde menciona que la idea de educación continua es una formación más práctica, esta circunstancia admite un mayor interés en los participantes. Esta percepción se refiere a lo esencial de la praxis en este tipo de programas. Además otra de las causas es que los profesionales en actividad van perdiendo su habilidad para resolver los problemas que se les presentan frente a tantos cambios y nuevas generaciones de profesionales con conocimientos actualizados. Por lo tanto, un aprendizaje continuo implica estar siempre actualizado y ser más competitivos al mundo actual (Metro Ecuador, 2019).

Los datos en las áreas de los procesos de educación continua presentan una demanda liderada por la categoría de Docencia y seguida de las 
Análisis documental relacionado con la educación continua como eje integrador de las competencias del currículo universitario

luis Danilo Flores Rivera, Carlos Fernando Meléndez Tamayo y Manuel Morocho

AMAGUAYA

de Investigación y Tecnologías, siendo las Específicas que se encuentran en el último lugar, registros que se limitan y responden a necesidades particulares relacionadas directamente con la prospectiva institucional. Así como la metodología acorde a los procesos que permiten desarrollar las competencias profesionales; pero porque no decirlo las competencias personales con la formación en habilidades blandas.

\section{Conclusiones}

Es claro que la actualización de conocimientos, perfeccionamiento de aprendizajes profesionales y las innovaciones metodológicas, determinan a la educación continua como el eje integrador de las competencias del currículo universitario. Uno de los componentes más importantes en el proceso de formación continua son las TIC, recurso que facilita y flexibiliza las modalidades de aprendizaje.

El crecimiento de la oferta en la educación continua en la Universidad permite proyectar cursos (dimensionar espacios físicos, capacidad tecnológica y seleccionar talento humano) que se ajusten a los requerimientos modernos y de las necesidades de la sociedad. Otro factor a considerar es la estructuración de modalidades, metodologías, técnicas y estrategias de capacitación y/o perfeccionamiento que se acondicionen al espacio-tiempo de los partícipes del proceso formativo. Sugerencia apoyada por los participantes de los cursos realizados y de la eventualidad académica.

Las IES en Ecuador están actualizando sus programas y perfiles académicos (rediseños curriculares) de acuerdo a las necesidades existentes y escenarios profesionales en los que posiblemente se desempeñaran los futuros profesionales. Estas acciones han permitido, que la educación continua trabaje de manera paralela facilitando y complementando nuevas competencias útiles para el mercado laboral. Por tanto, las empresas y las universidades no solo deben trabajar en conjunto, sino manejar una red de información y conocimientos respaldados por las TIC, que permiten ser un canal de comunicación para los procesos de actualización e intercambio tecnológico; con el fin de cumplir con las necesidades del personal y con los requerimientos de las organizaciones.

El crecimiento de la oferta de horas de capacitación es un nexo de los procesos de educación continua que certifican la competencia cursada y 
Análisis documental relacionado con la educación continua como eje integrador de las competencias del currículo universitario

luis Danilo Flores Rivera, Carlos Fernando Meléndez Tamayo y Manuel Morocho

AMAGUAYA

que el partícipe tenga la seriedad y el compromiso de aprender a aprender. Por lo que las IES deben brindar un abanico de posibilidades en las modalidades de educación continua combinadas a las competencias $T I C$, con el propósito de tener un mayor número de participantes que se favorezcan e integren a los nuevos enfoques de la gestión organizacional.

El crecimiento del número de participantes en los procesos de formación continua, tienen que ver con el modelo de la oferta académica (modalidad, competencias y contenidos curriculares), oportunidad de desarrollo y el interés motivado del beneficio que se genera para el participante y la organización, con resultados de crecimiento personal y profesional.

La experiencia institucional ha fomentado procesos de educación continua en Docencia, Investigación, Tecnologías y Específicas. Actualmente es considerada la solicitud de organizaciones en procesos de capacitación y desarrollo en habilidades blandas (Entrenamiento "Coaching", Liderazgo, trabajo en equipo, resolución de conflictos). Los académicos en general buscan áreas de tecnologías, investigación (publicación de artículos, libros), así como programas de educación inclusiva para personas con discapacidad y/o habilidades especiales.

\section{Referencias}

Andrade-Paco, J., Nava-Ortega, M. J., y Valverde-Núñez, J. (10 de Septiembre de 2009). La educación continua como proceso de formación académica en los alumnos egresados de las instituciones de educación superior en el estado de Sonora (México). Contabilidad y Negocios, 57-62. Recuperado el 28 de Noviembre de 2019, de http:// www.redalyc.org/articulo.oa?id=281621776008

Area-Moreira, M., y Adell-Segura, J. (2009). e-Learning: Enseñar y Aprender en Espacios Virtuales. En J. De Pablos (Coord.), Tecnología educativa. La formación del profesorado en la era de Internet (págs. 391-424). Málaga: Aljibe.

Arredondo-Galván, V. M. (2 de Enero de 2012). publicaciones.anuies.mx. Obtenido de http://publicaciones.anuies.mx/pdfs/revista/Revista81_S1A7ES.pdf

Arteaga-Linzán, M. M., López-Padrón, A., y Ruiz-Arnaud, J. R. (Mayo-Agosto de 2018). La capacitación y perfeccionamiento pedagógico de los docentes de la carrera de Medicina Veterinaria de la Universidad Técnica de Manabí: estudio diagnóstico de su estado actual. Cubana Educación Superior, 37(2), 78-86. Obtenido de http://www. rces.uh.cu/index.php/RCES/article/view/214/256

Barros-Morales, R., González-González, N., Pírela-Añez, A., Galindo-Mosquera, S., Rizzo-Bajaña, P., y Hidalgo-Tamayo, G. (2019). Gestión del Conocimiento Perspectiva Multidisciplinaria (Vol. XII). Zulia: Fondo Editorial Universitario de la Universidad 
Análisis documental relacionado con la educación continua como eje integrador de las competencias del currículo universitario

luis Danilo Flores Rivera, Carlos Fernando Meléndez Tamayo y Manuel Morocho

AMAGUAYA

Nacional Experimental Sur del Lago Jesús María Semprúm. Obtenido de http://www. unesur.edu.ve/libros/item/319-libro-gestion-el-conocimiento-perspectiva-multidisciplinaria-volumen-12

Beneitone, P., Esquetini, C., González, J., Marty Maletá, M., Siufi, G., y Wagenaar, R. (2007). Reflexiones y perspectivas de la Educación Superior en América Latina Informe Final - Proyecto Tuning - América Latina 2004-2007. (P. Beneitone, C. Esquetini, J. González, M. Marty Maletá, G. Siufi, y R. Wagenaar, Edits.) Bilbao: Universidad de Deusto. Obtenido de http://tuning.unideusto.org/tuningal/index.php?option=com_ docman\&Itemid=191\&task=view_category\&catid=22\&order=dmdate_ published\&ascdesc $=$ DESC

Bermúdez-Carrillo, L. A. (Enero de 2015). Capacitación: Una herramienta de fortalecimiento de las PYMES. InterSedes: Revista de las Sedes Regionales, XVI(33), 1-25. Obtenido de https://www.redalyc.org/articulo.oa?id=66638602001

Cabero-Almenara, J., De la Horra-Villacé, I., y Sánchez-Bolado, J. (2018). La realidad aumentada como herramienta educativa. Madrid, España: Paraninfo.

Cabrera-Núñez, G. (2016). Educación Superior, Formación Docente, Capacitación Continua y Calidad Educativa. El Caso de Baja California, México. I Congreso online sobre La Educación en el Siglo XXI, 417-427. Obtenido de http://www.eumed.net/ libros-gratis/actas/2016/educacion/gcn.pdf

Catalano, A., Avolio de Cols, S., y Sladogna, M. (2004). Diseño curricular basado en normas de competencia laboral. Buenos Aires: Banco Interamericano de Desarrollo.

Cejas-Martínez , M., y Acosta, J. A. (2012). La capacitación laboral: alcances y perspectivas en tiempos complejos. Anuario, 35, 148-173. Obtenido de http://servicio.bc.uc. edu.ve/derecho/revista/idc35/art06.pdf

CEPP, y GIZ. (2019). Programa de Educación Continua "Gestor de Desarrollo Endógeno Sostenible". Bases Conceptuales y Currículo. Quito: CEPP. Obtenido de http://ceppecuador.org/guardianes/talleres/Varios/ProgramaEducacionContinuaGDES.pdf

Chanta-Martínez, R. A. (2017). La educación con enfoque por competencias: ¿una oportunidad para impulsar la investigación en la universidad? Diá-logos, 39-49. Recuperado el 11 de Diciembre de 2019, de https://www.researchgate.net/publication/321811850_La_educacion_con_enfoque_por_competencias_una_oportunidad_ para_impulsar_la_investigacion_en_la_universidad

Chiavenato, I. (2011). Administración de Recursos Humanos. El capital humano de las organizaciones (Novena ed.). México, México: McGraw-Hill.

Consejo de Educación Superior. (22 de Enero de 2013). Consejo de Educación Superior. Obtenido de www.ces.gob.ec/doc: http://www.ces.gob.ec/doc/regimen_ academico/2da_ronda_de_talleres/Mesa3/gestin_curricular_completa.pdf

Consejo de Educación Superior. (21 de Marzo de 2019). Reglamento de Régimen Académico. Quito, Pichincha, Ecuador: Consejo de Educación Superior. Recuperado el 9 de Diciembre de 2019, de http://upse.edu.ec/secretariageneral/images/archivospdfsecretaria/1.\%20NORMATIVA\%20GENERALES/REGLAMENTO\%20DE\%20REGIMEN\%20ACADEMICO.pdf

DEaDV. (10 de Enero de 2020). https://deadv.uta.edu.ec. Obtenido de Dirección de Educación Continua a Distancia y Virtual: https://deadv.uta.edu.ec/index.php 
Análisis documental relacionado con la educación continua como eje integrador de las competencias del currículo universitario

luis Danilo Flores Rivera, Carlos Fernando Meléndez Tamayo y Manuel Morocho

AMAGUAYA

DEaDV. (24 de Enero de 2020). Reportes anuales de los cursos de educación continua. Ambato, Tungurahua, Ecuador. Recuperado el 3 de Febrero de 2020.

Eliot-Levine, S. P. (7 de Noviembre de 2019). iNACOL. Recuperado el 15 de Diciembre de 2019, de https://www.inacol.org/wp-content/uploads/2019/11/what-is-competency-based-education-an-updated-definition.pdf

Ferrés, J., y Piscitelli, A. (1 de Marzo de 2012). La competencia mediática: propuesta articulada de dimensiones e indicadores. Comunicar, XIX(38), 75-82. doi:http://dx.doi. org/10.3916/C38-2012-02-08

Flores-Rivera, L. D., y Meléndez-Tamayo, C. F. (30 de Junio de 2017). Variación de la autonomía en el aprendizaje, en función de la gestión del conocimiento, para disminuir en los alumnos los efectos del aislamiento. RED. Revista de Educación a Distancia, 1-15. Obtenido de https://www.um.es/ead/red/54/flores_melendez.pdf

Flores-Zapata, G., y Aballe-Pérez, V. C. (2018). La urgente necesidad de la capacitación y profesionalización del docente universitario. Estudios del Desarrollo Social: Cuba y América Latina, 6(3), 126-131. Obtenido de http://www.revflacso.uh.cu/index.php/ EDS/article/view/289/292

Gimeno-Sacristán, J., Pérez-Gómez, Á. I., Martínez-Rodríguez, J. B., Torres-Santomé, J., Angulo-Rasco, F., y Álvarez-Méndez, J. M. (2008). Educar por competencias, ¿qué hay de nuevo? Madrid: Morata, S.L.

Gómez, J. M. (11 de Noviembre de 2019). Análisis de pertinencia en el rediseño de carreras universitarias. Riobamba, Ecuador.

González, J., y Wagenaar, R. (2006). Informe Final Proyecto Piloto - Fase 2 Tuning Educational Structures in Europe II La contribución de las universidades al Proceso de Bolonia. (J. González, y R. Wagenaar, Edits.) Bilbao, España: Universidad de Deusto. Obtenido de http://www.deusto-publicaciones.es/deusto/pdfs/tuning/tuning04.pdf

González, V., y González, R. (2008). Competencias genéricas y formación profesional: un análisis desde la docencia universitaria. Iberoamericana de Educación, 47, 185209.

Grijalva-Verdugo, A. A., y Lara-Rivera, J. A. (2019). Competencias mediáticas en jóvenes universitarios. Análisis de saberes para producir contenido digital en una IES mexicana. Edutec. Revista Electrónica De Tecnología Educativa(67), 16-30. doi:https://doi. org/10.21556/edutec.2019.67.1297

Grijalva-Verdugo, A. A., y Urrea-Zazueta, M. L. (13 de Noviembre de 2017). Competencia digital y mediática. panorama socioeducativo del campo en México. Tecnologías de la información y la comunicación (TIC) en educación (págs. 1-11). San Luis Potosí: Consejo Mexicano de Investigación Educativa (COMIE). Recuperado el 15 de Febrero de 2020, de http://www.comie.org.mx/congreso/memoriaelectronica/v14/ doc/2732.pdf

Huerta, M. (2014). Formación por competencias a través del aprendizaje estratégico. Lima: San Marcos.

Huerta-Rosales, M., Penadillo-Lirio, R., y Kaqui-Valenzuela, M. (2017). Construcción del currículo universitario con enfoque por competencias Una experiencia participativa de 24 carreras profesionales de la UNASAM. Iberoamericana de Educación, 74, 83106. Obtenido de https://rieoei.org/historico/documentos/rie74a03.pdf 
Análisis documental relacionado con la educación continua como eje integrador de las competencias del currículo universitario

luis Danilo Flores Rivera, Carlos Fernando Meléndez Tamayo y Manuel Morocho

AMAGUAYA

Izquierdo-García, B., y Schuster-Fonseca, J. (19 de Agosto de 2009). www.uv.mx/iiesca. Obtenido de https://www.uv.mx/iiesca/files/2013/01/continua2000.pdf

Jamaica-González, F. M. (2016). Los beneficios de la capacitación y el desarollo del personal de las pequeñas empresas. Universidad Militar Nueva Granada, 1-18. Obtenido de https://repository.unimilitar.edu.co/bitstream/ handle/10654/7168/Trabajo\%20final\%20Fabian\%20Jamaica\%20\%281\%29. pdf? sequence $=1$ \&isAllowed $=y$

Junta de Andalucía. (13 de Marzo de 2015). juntadeandalucia.es/. Obtenido de http:// www.juntadeandalucia.es/averroes/centros-tic/14600929a/helvia/sitio/upload/Documento_base_modulo_1.pdf

Mateus, J. (28 de Mayo de 2018). Educación Mediática. Recuperado el 14 de Febrero de 2020, de http://educared.fundaciontelefonica.com.pe/educacion-mediatica/competencia/

Meléndez-Tamayo, C. F., y Flores-Rivera, L. D. (28 de Diciembre de 2018). Educación Continua, Gestor del Aprendizaje y Conocimiento en la Educación Superior. 3C TIC. Cuadernos de desarrollo aplicados a las TIC,, VII(4), 76-97. Obtenido de https://search.proquest.com/openview/559a462a4d05d51f204f21934a868b4c/1? pq-origsite $=$ gscholar $\&$ cbl $=2042722$

Metro Ecuador. (24 de Julio de 2019). ¿Por qué es tan importante la Educación Continua en el mundo actual? (N. Proaño, Ed.) metroecuador.com.ec. Obtenido de https:// www.metroecuador.com.ec/ec/noticias/2019/07/24/por-que-es-tan-importante-laeducacion-continua-en-el-mundo-actual.html

Ministerio de Educación y Formación Profesional - Gobierno de España. (2018). El currículo. Recuperado el 15 de Febrero de 2020, de https://www.educacionyfp.gob.es/ educacion/mc/lomce/el-curriculo/curriculo-primaria-eso-bachillerato/competenciasclave/competencias-clave/digital.html

Moreno-Olivos, T. (2010). El currículo por competencias en la universidad: más ruido que nueces. Revista de la Educación Superior, 77-90. Obtenido de http://www.scielo. org.mx/scielo.php?script=sci_arttext\&pid=S0185-27602010000200004

Morin, E. (1999). Los siete saberes necesarios para la educación del futuro. París: UNESCO. Obtenido de http://www.ideassonline.org/public/pdf/LosSieteSaberesNecesariosParaLaEdudelFuturo.pdf

Nares-González, M. L., y Soto-González, E. (2014). Formación y capacitación docente en las instituciones de educación superior y el impacto en la calidad educativa. Iberoamericana para la Investigación y el Desarrollo Educativo(12), 1-15. Obtenido de http://dspace.uan.mx:8080/jspui/bitstream/123456789/551/1/Formación\%20 y\%20capacitación\%20docente $\% 20$ en $\% 20$ las $\% 20$ instituciones $\% 20$ de $\% 20$ educación\%20superior\%20y\%20el\%20impacto\%20en\%20la\%20calidad\%20educativa.pdf

Ortega, S. (2 de Agosto de 2011). unesco.org. Obtenido de http://www.unesco.org/new/ fileadmin/MULTIMEDIA/FIELD/Santiago/pdf/Sylvia-Ortega-Formacion-Continua-Estrategia-Docente.pdf

Ortiz-Ocaña, A. (2014). Currículo y didáctica. Bogotá: Ediciones de la U. Obtenido de https://www.researchgate.net/publication/315835071 
Análisis documental relacionado con la educación continua como eje integrador de las competencias del currículo universitario

luis Danilo Flores Rivera, Carlos Fernando Meléndez Tamayo y Manuel Morocho

AMAGUAYA

Pailos, N. (11 de Octubre de 2019). America-retail.com. Obtenido de https://www.america-retail.com/opinion/opinion-beneficios-de-la-capacitacion-y-el-desarrollo-de-losrrhh-con-alta-tecnologia/

PRODEM. (1 de Enero de 2004). Empresa PRODEM. (P. Flores-Sepúlveda, Ed.) Obtenido de http://www.prodemsa.net/images/portal/articulos/CBC.pdf

Puntunet-Bates, M., y Domínguez-Bautista, A. (26 de Diciembre de 2008). La educación continua y la capacitación del profesional de enfermería. Revista Mexicana de Enfermería Cardiológica, XVI(3), 115-17. Recuperado el 1 de Diciembre de 2019, de https://www.medigraphic.com/pdfs/enfe/en-2008/en083g.pdf

Red Universitaria de Educación Continua. (2017). https://rededucacioncontinua.cl/. Recuperado el 15 de Diciembre de 2019, de https://rededucacioncontinua.cl/definicion-de-educacion-continua/

Rico, L. (2004). Análisis conceptual e investigación en didáctica de la matemática. EMA, IX(1), 3-19. Obtenido de http://funes.uniandes.edu.co/1508/1/109_Rico2004Análisis_RevEMA.pdf

Rivadeneyra-Espinoza, L., Rivera-Grados, D., Sedeño-Monge, V., López-García, C., y Soto-Vega, E. (2016). La capacitación del profesorado universitario. Tendencias pedagógicas, 28, 45-54. Obtenido de https://revistas.uam.es/tendenciaspedagogicas/ article/viewFile/3733/4947

Sánchez. (2012). Educación continua en la Universidad de Chile. Primer Encuentro de Educación Continua de la Universidad de Chile: Realidades y Perspectivas (págs. 1-7). Santiago de Chile: Universidad de Chile. Recuperado el 7 de Diciembre de 2019, de https://www.uchile.cl/documentos/sobre-la-educacion-continua-pdf-509kb_124867_0_4840.pdf

Tobón, S., Pimienta, J., y García, J. (2012). Secuencias didácticas: Aprendizaje y evaluación de competencias. México: Pearson Educación.

Tobón-Tobón, S., Pimienta-Prieto, J. H., y García-Fraile, J. A. (2010). Secuencias didácticas: aprendizaje y evaluación de competencias. México: Pearson Educación. Obtenido de https://www.researchgate.net/publication/287206904

Tuning. (2007). Reflexiones y perspectivas de la educación superior en América Latina. Bilbao: Fondo Editorial de la Universidad de Deusto.

UNESCO. (13 de Julio de 2018). Sistemas de Información de Tendencias Educativas en América Latina. Obtenido de SITEAL: http://www.siteal.iipe.unesco.org/sites/default/ files/sit_accion_files/siteal_mexico_0097.pdf

Universidad ESAN. (19 de Septiembre de 2016). Conexión ESAN. Obtenido de Apuntes Empresariales: https://www.esan.edu.pe/apuntes-empresariales/2016/09/la-capacitacion-y-el-desarrollo-de-competencias/

Valverde-Berrocoso, J. e. (2019). Campus digitales en la Educación Superior. Experiencias e investigaciones. (S. d. Extremadurac, Ed.) Extremadura: Universidad de Extremadura. Obtenido de http://hdl.handle.net/10662/8658

Verdezoto-Rodríguez, R. H., y Chávez-Vaca, V. A. (2018). Importancia de las herramientas y entornos de aprendizaje dentro de la plataforma e-learning en las Universidades del Ecuador. EDUTEC. Revista Electrónica de Tecnología Educativa, 65, 68-90. doi:https://doi.org/10.21556/edutec.2018.65.1067 
Análisis documental relacionado con la educación continua como eje integrador de las competencias del currículo universitario

luis Danilo Flores Rivera, Carlos Fernando Meléndez Tamayo y Manuel Morocho

AMAGUAYA

Zabalza, M. (2008). Los planes de estudio en la universidad. Algunas reflexiones para el cambio. Santiago de Compostela: Universidad de Santiago de Compostela.

Zapata-Guerrero, E. E. (2012). Mercadeo Educativo: Estrategias para Promover Instituciones y Programas (Segunda ed.). Bógota: ePub X Publidisa. 